Journal of Animal and Veterinary Advances 11 (4): 509-516, 2012

ISSN: $1680-5593$

(C) Medwell Journals, 2012

\title{
Genetic Variation and Population Structure of Thai Indigenous Pig Populations Based on Mitochondrial and Microsatellite DNA Markers
}

\author{
${ }^{1,2}$ S.L. Yang, ${ }^{2}$ B. Surintorn, ${ }^{2}$ N.L. Pongchan, ${ }^{3}$ N.N. Uthairat and ${ }^{4}$ Z.H. Shi \\ ${ }^{1}$ Department of Animal Science, Guizhou University, Guiyang, China \\ ${ }^{2}$ School of Animal Production Technology, Suranaree University of Technology, \\ Nakhon, Ratchasima, Thailand \\ ${ }^{3}$ Fish Genetic Laboratory, Faculty of Fisheries, Kasetsart University, Bangkok, Thailand \\ ${ }^{4}$ Animal Husbandry Bureau of Zunyi, Guizhou Province, China
}

\begin{abstract}
To understand molecular genetic characteristics of Thai indigenous pig populations, researchers compared the genetic variation at 12 microsatellite loci and the mitochondrial DNA $C y t b$ gene in two populations of Thai indigenous pigs including Northeast Thai (NT) and South Thai (ST) pigs, a Wild Boar (WB) population and a Chinese Qianbei Black (CQB) pig population from Guizhou province of China. The higher effective number of alleles were detected in NT (7.09) and ST (6.3) pig compared to CQB (5.97) and WB (5.19) pigs. NT pigs had the highest PIC value (0.82) among all populations. The average expected Heterozygosity (HE) was 0.72 in NT and 0.71 in ST and 0.69 in WB pigs which was lower than that observed in CQB pigs (0.82). Hardy-Winberg (HWE) test showed of all 12 microsatellite loci, 7 loci were deviated from HWE ( $\mathrm{p}<0.05$ ). Seventy four polymorphism sites were detected among $35 \mathrm{Cyt} b$ gene sequences of $1046 \mathrm{bp}$ in four populations. Eighteen Cyt b haplotypes were detected and haplotype H9 was found in CQB, NT and ST pigs. Both the $\mathrm{F}_{\mathrm{ST}}$ and Nei 's standard genetic Distances (Ds) from the microsatellite data indicated genetic differentiation between Thai pigs and Chinese Southwest pigs but UPGMA tree showed that Thai pigs had close relationship with Chinese pigs. Structure analysis indicated that NT populations were identified as the separate clusters while few $\mathrm{ST}$ individuals was assigned into the $\mathrm{CQB}$ population at $\mathrm{K}=2$. Median-joining network analysis showed that the NT population constituted predominated haplogroups (54.28\%). These results suggest that Thai indigenous populations have high genetic diversity in both nuclear and mtDNA data. The close relationship between Chinese Southwest pigs and Thai pigs implies that Thai native pigs might have the same origin as pigs from South or Southwest China. These findings can be used as valuable genetic information for the preservation and further genetic improvement of the Thai indigenous pig population.
\end{abstract}

Key words: Microsatellite, mtDNA Cyt b, Thai indigenous pig, genetic diversity, improvement, China

\section{INTRODUCTION}

The Thai pig industry became increasingly commercialized in the 1980 s. Since, that time, village production of pigs has declined and commercial units have become the main source of supply of pork in Thailand (Tisdell and Harrison, 1997). Accordingly, the number of Thai indigenous pigs has been rapidly decreasing since exotic pig breeds were introduced for breeding improvement. Thai indigenous pigs are classified into several different phenotypic groups distributing in the Northern and the Southern areas of Thailand (Kazue, 1974). Some of Thai wild boars (Sus scrofa jubatus) were crossed with Chinese Meishan pig for commercial requirements. These indigenous pig populations are genetically adapted to endure food and water scarcity and resist extreme tropical disease and climate conditions. Although, some pigs have the same appellations as Chinese native pigs, there are no evidences that whether these Thai pigs have the common ancestors with Chinese pigs. The classification for Thai pigs is based on historic appellations and phenotypic characteristics. However, environmental factors have probably influenced phenotypic characteristics, so this classification is no longer inappropriate for the formulation of breed improvement and utilization strategies (Dadi et al., 2008). The analysis of genomic DNA variation among and within populations allows the optimum utilization of animal genetic resources and permits efficient genetic improvement for production and conservation needs.

Corresponding Author: Shenglin Yang, Department of Animal Science, Guizhou University, 550025 Guiyang, Guizhou Province, China 
Effective species conservation programs require the evaluation of intra-species genetic diversity (Haig, 1998). Although, genetic relationship among several pig populations including Thai indigenous pigs in East Asia has been analysed by blood groups and serum protein polymorphism (Tanaka et al., 1983), no studies have been reported on the genetic diversity and population structure of Thai Indigenous pigs using mitochondrial and microsatellites DNA markers. Information on Thai indigenous pigs is also very scarce and to the knowledge, limited to one report in which genetic variation of the Thailand indigenous pig populations were analysed using microsatellite markers (Chaiwatanasin and Chantsavang, 2002). In this study, researchers investigated genetic variation and structure of several Thai pig populations using sequence data from the mitochondrial $C y t b$ gene and 12 microsatellite loci.

\section{MATERIALS AND METHODS}

Samples and DNA extraction: The study samples comprised 112 individuals represent ing the four pig populations distributed in Thailand and China: Northeast Thai pig (NT, $\mathrm{n}=61$ ), Nouth Thai pig ( $\mathrm{ST}, \mathrm{n}=23$ ) wild boar ( $W B, n=7)$, Chinese Qianbei black pig (CQB, $N=21$ ) (Fig. 1). NT samples were collected from Northeastern Thailand including the following six provinces: Sakorn Nakhon, Nakorn Panom, Mokdahan, Si Saket, Loei and Surin. All hair samples were taken from farmer's backyards in these provinces. ST samples were collected from Nakorn Si Thamamarat province, one of the representative provinces in Southern Thailand. WB samples were also gathered from Northeastern Thailand and number of individuals sampled in this population was quite low due to sampling difficulties and inconveniences. In addition, CQB samples were collected from Chinese Qianbei Black Pig Conservation Farm in Zunyi city, Guizhou province of China.

Wizard Genomic DNA Purification kit (Promega, Madison, WI) was used to extract DNA from hair roots samples. For 100 hair root samples, $10 \mu \mathrm{L}$ of proteinase $\mathrm{K}$ was added for each tube after adding $200 \mu \mathrm{L}$ Nucleic lysis solution.

Microsatellite analysis: Twelve primers were selected from 27 pairs of microsatellite primers in swine recommended by FAO/ISIG in 2004 and PCR were performed in $10 \mu \mathrm{L}$ reaction mixture containing $2.5-5.0 \mathrm{ng}$ template DNA, $1 \mu \mathrm{L}$ of $10 \times$ buffer $(10 \mathrm{~mm}$ Tris, $50 \mathrm{~mm} \mathrm{Kcl}$, $0.1 \%$ gelat in $\mathrm{pH} 8.4), 0.8 \mu \mathrm{L}$ of $2.5 \mathrm{mM}$ dNTP mixture, $1.5-4.0 \mathrm{mM} \mathrm{MgCl}_{2}(0.6-1.6 \mu \mathrm{L}), 1 \mu \mathrm{L}$ of $10 \mathrm{pmol}$ primer and $0.25 \mathrm{U}$ Taq DNA polymerase.

The amplification was performed in iCycler PCR system (BIO-RAD, USA) with an initial denaturation at $95^{\circ} \mathrm{C}$ for $5 \mathrm{~min}$ followed by 35 cycles of denaturation at $95^{\circ} \mathrm{C}$ for $30 \mathrm{sec}$, annealing at optimum temperature $55-62^{\circ} \mathrm{C}$ for $30 \mathrm{sec}$ and extension at $72^{\circ} \mathrm{C}$ for $30 \mathrm{sec}$ with a final extension at $72^{\circ} \mathrm{C}$ for $5 \mathrm{~min}$. PCR reactions were performed according to recommended annealing temperatures and concentrates of $\mathrm{MgCl}_{2}$ with suitable adjustments, so as to acquire the good quality of PCR products for running Polyacrylamide Gel Electrophoresis (PAGE). About $3 \mu \mathrm{L}$ denatured PCR products were loaded into $6 \%$ denaturing polyacrylamide sequencing gel, molecular marker Ph1 $\times 174 /$ Hinfl and sequencing makers M13 ladder were loaded into middle of each panel gel.

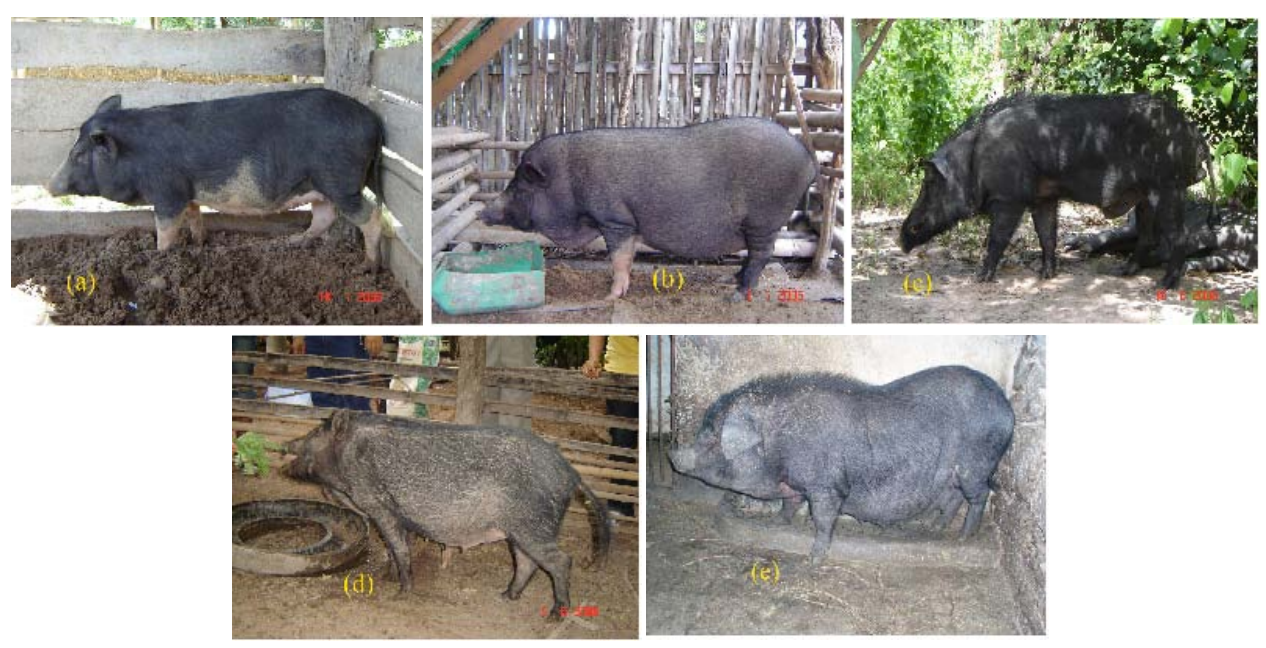

Fig. 1: Sample representatives; a) South Thai pig; b) Northeast Thai pig; c) wild pig (male); d) wild pig (female) and e) Chinese Qianbei black pig 
Preparations for M13 ladder solution, silver staining solution according to the methods descried by Promega Corporation. Scoring of microsatellite genotypes is straight forward.

mtDNA sequencing: The $1046 \mathrm{bp}$ fragment of $C y t b$ gene was amplified and sequenced in 35 individuals from three Thai pig population and one Chinese pig population $(\mathrm{NT}=19, \mathrm{ST}=7, \mathrm{CQB}, \mathrm{N}=7$ and $\mathrm{WB}=2$ ). To amplify the Cyt $b$ region, the primers mitL1 (5'-ATCGTTGTCATTC AACTACA-3' (Watanobe et al., 1999) in the L strand of the tRNA for glutamic acid and mitH2 (5'-CTCCTTCTCGG TTTACAAG-3') in the $\mathrm{H}$ strand of the tRNA for threonine were used. PCR were performed in iCycler PCR system (BIO-RAD, USA) with an initial cycle at $95^{\circ} \mathrm{C}$ for $30 \mathrm{sec}$ followed by 35 cycles at $95^{\circ} \mathrm{C}$ for $45 \mathrm{sec}$ and $55^{\circ} \mathrm{C}$ for $30 \mathrm{sec}$ and followed by $72^{\circ} \mathrm{C}$ for $1 \mathrm{~min} 72^{\circ} \mathrm{C}$ extension for $7 \mathrm{~min}$. PCR products were purified and directly sequenced by Macrogen Co., in South Korea using Big Dye Terminator v3. 1 Cycle Sequencing kit (Applied Biosystems).

Statistical analysis: For microsatellite, the numbers of alleles (No), effective number of alleles $(\mathrm{Ne})$, expected $(\mathrm{HE})$ and observed heterozgosity $(\mathrm{HO})$, allele frequencies were computed using GENEPOP 3.4 package (Raymond and Rousset, 1995) according to Kimura and Crow (1964), Polymorphism Information Content (PIC) values were calculated using little program PIC-CALC 0.6 (http://www. bbioo.com).

The classic Wright indices (FIT, FIS and $\mathrm{F}_{\mathrm{ST}}$ ) were calculated (Wright, 1965; Weir and Cockerham, 1984) using POPGENE Version 1.3 (Yeh et al., 1997). Hardy-Weinberg Equilibrium (HWE) was also tested using a $\chi^{2}$-test for each pair of locus/population by the Fisher's Method implemented in the GENEPOP 3.4 package.

Expected homozygosity and heterozygosity were computed according to Levene (1949), Nei's expected heterozygosity was computed according to Nei (1972)'s. Several diversity parameters were computed from mtDNA Cyt b sequences using ARLEQUIN 3.0 software (Excoffier and Schneider, 2005) including nucleotide and mtDNA haplotype diversity, measures of polymorphism as number of haplotypes, excusive haplotypes, number of polymorphic sites, nucleotide differences per sites, haplotype diversity and nucleotide diversity.

Genetic relationships among populations were established using three methods. First, the nuclear pairwise $\mathrm{F}_{\mathrm{ST}}$ were compared among the populations under the hypothesis that genetic drift is the main factor causing genetic differentiation between populations. Nei's standard distance (Nei, 1972) based on data of 12 microsatellite markers among the four populations were computed. An unrooted phylogenetic tree was also constructed using UPGMA Method (Sneath and Sokal, 1973 ) based on 12 microsatellite locus using PHYLIP Version 3.67 (Felsenstein, 1993). Bootstraps of 1000 replicates were performed in order to test the robustness of tree topology (Efron et al., 1996). Second, a Bayesian maximum likelihood approach to infer the number of populations without prior information of the sampling location was used through the software Structure 2.2 (Pritchard et al., 2000; Falush et al., 2003). This method estimated the proportion of the individual genome derived from each inferred ancestral population and the number of ancestral populations represented in the samples. The genetic cluster analysis was performed assuming an Admixture Model (Pritchard et al., 2000). Third, a network analysis was used to visualize the spatial distribution of sequence variation among the different mtDNA Cyt $b$ haplotypes using the program Network 4.2 (Bandlet et al., 1999). This procedure reveals possible evaluat ion pathways (Bandelt, 1995) and is based on a maximum parsimony procedure (Templeton et al., 1992).

\section{RESULTS}

Genetic diversity: The average effective number $(\mathrm{Ne})$ of alleles, the average expected Heterozygosity ( $\mathrm{HE}$ ) and PIC at 12 studied loci in four pig populations are shown in Table 1. The higher observed mean number of alleles and higher effective number of alleles were detected in NT $(10.75 ; 7.09)$ and ST $(9.92 ; 6.3)$ populations compared to $\mathrm{CQB}(9.17 ; 5.97)$ and $\mathrm{WB}(6.5 ; 5.19)$ populations. The PIC value for single population ranged from 0.77 (WB) to 0.82 (NT), a relatively low Ne value in WB population (5.19). The expected Heterozygosity (HE) was mainly considered because it is regarded as a better estimator of the genetic variability presented in a population. As shown in Table 1, the HE values ranged from 0.69 (WB) to 0.82 (CQB). Hardy-Winberg test showed of all 12 microsatellite loci, 7 loci were deviated from HWE $(\mathrm{p}<0.05$ ). In general, observed HWE deviations were not consistent; they occurred with different microsatellites in different populations.

FIS is an estimate of variation within populations that measures the reduction in heterozygosity in an individual due to nonrandom mating within its subpopulation. In present study, FIS parameter ranged from 0.03 (CQB) to 0.22 (WB) (Table 1) indicating CQB had lower inbreeding degree compared to wild boar population from Thailand. The closed FIS values for ST and NT populations indicated similar heterozygous genotypes. 
Table 1: Within-population diversity values and population structure derived from the microsatellite loci and mtDNA diversity estimates

\begin{tabular}{|c|c|c|c|c|c|c|c|c|c|}
\hline \multirow[b]{2}{*}{ Population } & \multirow[b]{2}{*}{ Locality } & \multirow[b]{2}{*}{ Sample size } & \multicolumn{4}{|c|}{ Microsatellite data } & \multicolumn{3}{|c|}{ mtDNA data } \\
\hline & & & $\mathrm{H}_{\mathrm{F}}$ & PIC & $\mathrm{N}_{\mathrm{e}}$ & $\mathrm{F}_{\mathrm{IS}}$ & $\mathrm{N}_{\mathrm{h}}$ & $\mathrm{h}$ & $\pi$ \\
\hline ST & South, Thailand & 23 & 0.71 & 0.81 & 6.30 & $0.16^{*}(6)$ & 4 & 0.714 & 0.0027 \\
\hline NT & Northeast, Thailand & 61 & 0.72 & 0.82 & 7.09 & $0.16^{*}(6)$ & 9 & 0.819 & 0.0053 \\
\hline WB & North, Thailand & 7 & 0.69 & 0.77 & 5.19 & $0.22(1)$ & 2 & 1.000 & 0.0009 \\
\hline $\mathrm{COB}$ & Guizhou, China & 21 & 0.82 & 0.79 & 5.97 & $0.03(4)$ & 6 & 0.952 & 0.0045 \\
\hline
\end{tabular}

$\mathrm{H}_{\mathrm{E}}=$ Expected heterozygosity, PIC $=$ Mean Polymorphic Information Content, $\mathrm{N}_{\mathrm{e}}=$ Effective number of alleles, $\mathrm{F}_{\mathrm{IS}}=$ Inbreeding coefficient; $\mathrm{N}_{\mathrm{h}}=$ Number of haplotype, $\mathrm{h}=$ Haplotype diversity, $\pi=$ Nucleotide diversity; CQB $=$ Chinese Qianbei Black pigs, $\mathrm{ST}=\mathrm{South}$ Thai pigs, NT $=$ Northeast Thai pigs; WB $=$ Wild Boars; Number in parentheses indicates the number of loci showing a significant departure $(p<0.05)$ from Hardy-Weinberg equilibrium after correction for multiple tests

Table 2: Characterization of the 12 microsatellites analyzed in four pig populations

\begin{tabular}{lrcc}
\hline LocuS & $\mathrm{F}_{\text {IS }}$ & $\mathrm{F}_{\text {IT }}$ & $\mathrm{F}_{\text {ST }}$ \\
\hline S0227 & 0.21 & 0.28 & 0.09 \\
S0090 & 0.26 & 0.34 & 0.11 \\
S0226 & -0.05 & 0.01 & 0.05 \\
S0005 & 0.25 & 0.29 & 0.06 \\
S0068 & 0.04 & 0.13 & 0.09 \\
S0225 & 0.12 & 0.20 & 0.09 \\
S0155 & 0.05 & 0.13 & 0.09 \\
SW122 & 0.02 & 0.09 & 0.08 \\
S0355 & -0.08 & 0.04 & 0.11 \\
S0386 & 0.29 & 0.36 & 0.10 \\
SW911 & -0.11 & -0.01 & 0.09 \\
SW24 & 0.09 & 0.19 & 0.11 \\
Mean & 0.09 & 0.17 & 0.09 \\
\hline
\end{tabular}

$\mathrm{F}_{\mathrm{ST}}$ is measures of the genetic differentiation over subpopulations. Bonferroni correction $(p<0.05 / 12=0.0041) * p<0.05$

About 74 polymorphism sites were detected from $35 \mathrm{Cyt} b$ gene sequences of $1046 \mathrm{bp}$ in four populations, there were 62 transitions and 12 transversions, no insertions and deletions were observed. Estimates of mtDNA genetic diversity are shown in Table 1 .

About 18 mtDNA haplotypes were detected and denoted by $\mathrm{H} 1-\mathrm{H} 18$ showing a high genetic maternal diversity within and among the populations. Among all the haplotypes, H1-H9 were produced from NT population, H9 was found not only in $\mathrm{CQB}$ pigs but in NT and ST populations, $\mathrm{H} 8$ was detected both in NT and WB populations, the remaining haplotypes such as H1-H7_CH10-H18 occurred only in single population.

Among the all populat ions analysed, NT had 9 haplotypes and higher diversity for all estimates ( $\mathrm{h}=$ $0.0819, \pi=0.0053$ ) while WB had only 2 haplotypes and the lowest diversity ( $h=1 \pi=0.0009$ ) because only two Cyt $b$ sequences of WB samples were analysed and two independent haplotypes were detected for this population.

Genetic differentiation and relationships among populations: The statistic $\mathrm{F}_{\mathrm{ST}}$ an estimate of variation due to differences among populations which is the reduction in heterozygosity of a subpopulation due to genetic drift. Wright's F-statistic ( $\mathrm{F}_{\mathrm{ST}}, \mathrm{F}$ and FIS ) values for each locus are shown in Table 2 . The $\mathrm{F}_{\mathrm{ST}}$ perlocus varied from 0.05 (S0226)-0.11 (S0090, S0355, SW24) and the average $\mathrm{F}_{\mathrm{ST}}$ of all loci was 0.09. Multilocus $\mathrm{F}_{\mathrm{ST}}$ values indicated that around $9 \%$ of the total genetic variation was explained by
Table 3: Nuclear Pair-wise $F_{\mathrm{ST}}$ values (below the diagonal) and Nei's standard genetic distances (above the diagonal) among 4 pig populations

\begin{tabular}{lllll}
\hline Pig population & ST & NT & WB & CQB \\
\hline ST & - & 0.71 & 0.76 & 0.95 \\
NT & $0.06^{* * *}$ & - & 0.51 & 0.98 \\
WB & $0.06^{* * *}$ & $-0.01^{* * *}$ & - & 0.99 \\
CQB & $0.12^{* * *}$ & $0.07^{* * *}$ & $0.04^{* *}$ & - \\
\hline
\end{tabular}

Nei's $D_{\mathrm{A}}(\mathrm{Nei}, 1983)$ and pairwise $\mathrm{F}_{\mathrm{ST}}$ (Weir and Cockerham, 1984) are measures of genetic distance and genetic differentiation between populations, respectively; *** $p<0.01$ from a multi-locus test that there is no genetic differentiation in population pair after corrections for multiple comparisons

population differences with the remaining $91 \%$ corresponding differences among individuals within population.

Population genetic analysis showed no evidence for genetic differentiation between $\mathrm{CQB}$ and $\mathrm{WB}\left(\mathrm{F}_{\mathrm{ST}}=0.04\right.$; $\mathrm{p}=0.07)$ and also between NT and WB populations $\left(\mathrm{F}_{\mathrm{ST}}=-0.01 ; \mathrm{p}=0.81\right.$ ) (Table 3 ). However, significant differentiation was found between the ST and CQB, NT and $\mathrm{CQB}, \mathrm{NT}$ and $\mathrm{ST}, \mathrm{WB}$ and $\mathrm{ST}$ populations $\left(\mathrm{F}_{\mathrm{ST}}=0.12 ; \mathrm{F}_{\mathrm{ST}}=0.07, \mathrm{~F}_{\mathrm{ST}}=0.06, \mathrm{~F}_{\mathrm{ST}}=0.06\right.$, respectively $\mathrm{p}<0.001)$.

Nei's standard genetic distances among four populations are shown in Table 3 . $\mathrm{CQB}$ and WB had the largest distance while NT and WB had the smallest. Nei's unbiased genetic distance was also measured considering that a comparatively smaller pig population. A similar result could be obtained although, the absolute values of genetic distances were different from Nei's standard genetic distances. The UPGMA tree (Fig. 2a-c) was constructed based on the standard genetic distance. The numbers at the nodes are values for 1,000 bootstrap resampling of the 12 genotyped loci.

Graphical displays of the results from the structure analysis are shown in Fig. 2a, the most likely $\mathrm{K}$ value was that where in $\operatorname{Pr}(\mathrm{G} / \mathrm{K})$ is maximized. The maximum value of in $\operatorname{Pr}(\mathrm{G} / \mathrm{K})$ was acquired at $K=2$ suggesting that the most probable number of populations inferred was two. At $\mathrm{K}=2$, genetic population 1 (red) was mainly constituted from $\mathrm{CQB}$ individuals and a few of ST individuals while genetic population 2 (green) from NT and WB individuals. When $\mathrm{K}=3, \mathrm{CQB}$ and NT populations were identified as separate clusters while WB shared a small fraction with ST population. At $\mathrm{K}=4, \mathrm{CQB}$ and NT populations 


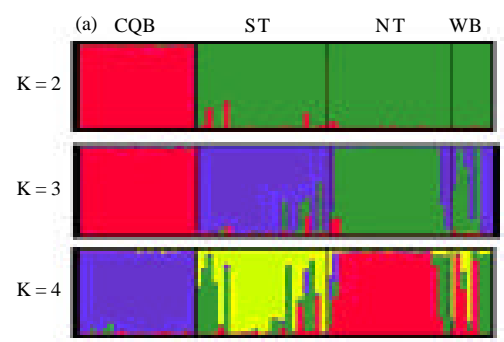

(b)

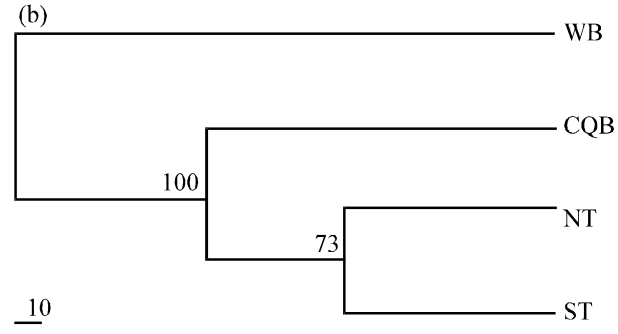

(c)

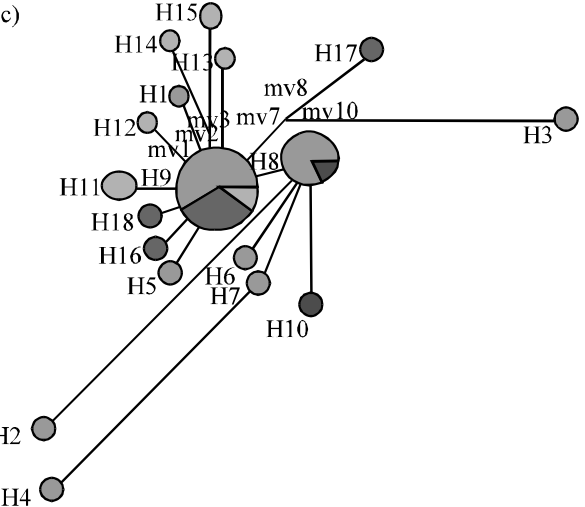

Fig. 2: a) Estimated membership fractions of pig populations for each of $\mathrm{K}$ inferred clusters ( $\mathrm{K}=2-4$ ) using the Structure program (Pritchard et al., 2000). Each individual was represented by a vertical line partitioned into $\mathrm{K}$ colors while $\mathrm{K}$ was the number of clusters assumed and the length of the colored segment represents the individul's estimated proportion of membership to a particular cluster; b) UPGMA tree showing the genetic relationships among four pig populations from Nei's standard distance (Nei, 1972) based on data of 12 microsatellite markers. The numbers at the nodes were percentage bootstrap values from 1,000 replications of re-sampled loci; c) Median-joining network analysis of eighteen mtDNA Cyt b haplotypes found in this study. Circles sizes were proportional to the observed frequency. Pig populations were defined based on geographical origins and were represented according to the following: south Thai pig (green), Northeast Thai pig (purple), Chinese Qianbei black pig (red), wild Boar (yellow) were still identified as the separate two clusters. Medianjoining network analysis showed the spatial distribution of eighteen haplotypes from $35 C y t b$ gene sequences (Fig. 2-c). The detection of 18 haplotypes in 35 Cyt b sequence suggested that abundant genetic diversity existed in Thai indigenous pig populations also in Chinese pig population. Thai indigenous populations did not cluster together and were rather interspersed among the rest of the sequences. Overall, the network spatial distribution tended to form three clusters and showed a starlike phylogenetic pattern in which two higher frequencies of haplotypes, $\mathrm{H} 9$ and $\mathrm{H} 8$ were located in the center of three clusters. The haplotypes from the NT population constituted predominated ones $(54.28 \%$, 19/35). Haplotypes from the ST and CQB populations were in $20 \%$, respectively while haplotype from WB was $5.7 \%$.

\section{DISCUSSION}

In this study, the mean allele number and the genetic diversity of Thai indigenous pig populations sampled from the Northeast to the South across the country was assessed. A Chinese CQB pig population was studied to compare the level of genetic diversity. The observed heterozygosities and expected heterozygosities were detected among four pig populations. Thai indigenous pigs exhibited moderate levels of allelic diversity and heterozygosity whereas Chinese Qianbei black pigs had a relatively high degree of genetic diversity. Moderate genetic diversity of Thai indigenous pigs is consistent with the previous report (Chaiwatanasin and Chantsavang, 2002). The levels of allelic diversity and heterozygosity of Thai pigs are similar to those reported for Chinese Southwest pigs (Fan et al., 2002; Fang et al., 2005) and Vietanmese pigs (Thuy et al., 2006). However, $\mathrm{HE}$ and $\mathrm{Ne}$ of Thai pigs were higher than that of Korean and European pigs (Kim et al., 2005; Laval et al., 2000; Li et al., 2004; Martinez et al., 2000; Lemus-Flores et al., 2001).

The Thai indigenous pig population showed relatively high levels of genetic diversity which is presumably to be a consequence of introducing exotic breeds to crossbreed with Thai pigs and lack of systematic artificial selection. In this study, all samples including NT, ST and WB populations were taken from peasants' backyards of villages and no intensive farms were found for these indigenous pigs. Randomly mating and non-artificial selection have been therefore, made for many years which may explain why Thai indigenous pig populations including $\mathrm{NT}$ and ST exhibited high $\mathrm{HE}$ levels ( $\mathrm{HE}=0.72,0.71$, respectively). This result could be 
illustrated by high PIC values in NT and ST populations. The HE value (0.82) in the Chinese Qianbei Black pig population was slightly higher than that of Chinese Rongchang pig populations reported by Fang et al. (2005). This may be due to less controlled mating and artificial selection. The lowest HE observed in WB pigs may due to a relatively small number of wild boars with inbreeding. Deviation from HWE in 7 microsatellite loci could be the result of the sampling of a single population with different allele frequencies in the subpopulations (Wahlund effect), nonrandom sampling, and/or inbreeding. In present study at least two populations, NT and ST, showed evidence of significant inbreeding (Table 1).

Among four pig populations, Chinese Qianbei Black pig population possessed the lowest FIS value (FIS $=0.03$ ) which may be due to randomly mating without the intensively artificial selection within the population. In contrast, wild boar population with the highest FIS value (FIS $=0.22$ ) could be on account of a relatively small population. WB individual was so scarce that it was quite difficult to acquire samples. Inbreeding was hard to avoid occurring within WB population. Hardy-Winberg test has shown no heterozygote excess in all loci in all populations $(\mathrm{p}<0.05)$. Deficiency of heterozygotes was probably caused by the Walhund effect which has been proposed in other domestic pigs such as Mexican hairless pig population (Lemus-Flores et al., 2001) and Iberian cattle breeds (Martin-Burriel et al., 1999). Throughout the 1960s and 1970 s Thai pig production was dominated by the backyard raising of pig cross-breeds for consumption. Since, 1980s, Thai pig industry became increasingly commercialized, village production of pigs has declined and commercial units have become the main source of supply of pork in Thailand (Murphy and Tisdell, 1995). Researchers found some Thai pig populations had similar callings with Chinese pigs during sampling process, a survey for this was made and result indicated that some Chinese breeds were introduced to Thailand in the middle of 20th century, these similar calling had been remained. This is the reason why CQB population was selected to be a sample in this research. From geographically speaking, Thailand is closed to the South and the Southwest of China where are possible regions for gene exchange. In fact, structure analysis may confirm this deduction to some extent because $\mathrm{CQB}$ had a low percentage assigned to the ST and NT populations (Fig. 2a).

Eighteen haplotypes could be detected from 35 $C y t b$ gene sequences, indicating that a high mtDNA genetic diversity existed in four pig populations. Proportion of haplotypes from Thai indigenous pigs including NT and ST populations reached to $74.28 \%$, reflecting that mtDNA polymorphism within these two populations is relatively high. It is worth mentioning that haplotype $\mathrm{H} 9$ was shared among $\mathrm{CQB}, \mathrm{NT}$ and ST populations, implying certain close correlation existed in these populations.

This inference also could be supported by a phylogenetic tree based on Nei's $D_{s}$ standard genetic distances using UPGMA method (Fig. 2b). NT was grouped into the same branches with ST population with a $73 \%$ of bootstrap support value. $\mathrm{CQB}$ and two Thai indigenous populations (NT, ST) were clustered into the same branches with a $100 \%$ of bootstrap support value but WB was clustered into another branch. This result is consistent with that from the structure analysis.

When deciding conservation priorities, several factors should be taken into account such as adaptation to specific environments or diseases, possession of special traits of cultural and scientific or future economic value (Ruane, 1999). Conservation decisions will depend on future plans for the breeds in question. If the purpose is to use them in crossbreeding or introgression plans, the diversity between subpopulations should be prioritized. However, if the purpose is to preserve a closed population capable to coping with future challenging environments or with diversified production conditions, the within-population diversity should be prioritized (Thirstrup et al., 2008). From the data, the $\mathrm{F}_{\mathrm{ST}}$ statistic revealed that a high significant level of genetic differentiation among the study populations although, a lack population differentiation was found between the two populations from WB (Chinese Qianbei Black and Northeast Thai pigs $)$ with nuclear markers $\left(\mathrm{F}_{\mathrm{ST}}=0.04\right.$ and $\mathrm{F}_{\mathrm{ST}}=-0.01$, respectively), estimation of the overall $\mathrm{F}_{\mathrm{ST}}$ in present study was 0.09 which was between European pig populations $\mathrm{F}_{\mathrm{ST}}=0.27$; Laval et al., 2000) and Chinese populations $\left(\mathrm{F}_{\mathrm{ST}}=0.077\right.$; Yang et al., 2003). Nevertheless, these differences are most likely to be explained by the different types of markers analysed as well as the source of population sampled.

\section{CONCLUSION}

In this study, Thai indigenous populations exhibited high genetic diversity in both nuclear and mtDNA information. However, Thai indigenous pig populations were distinct from Thai wild pig and Chinese Southwest pig populations. Haplotype network with mtDNA data showed that most of the Thai indigenous pig haplotypes were distinctly different from those of Chinese pig and Thai wild pig populations although, haplotype H9 was shared among Chinese Qianbei black pigs, Northeast Thai 
pigs and South Thai pigs. These findings suggested that the introgression might have been occurred between Chinese pig and Thai pig populations. Today, Thai indigenous pig populations are at risk of gradually losing their genetic diversity unless effective and appropriate breeding management practices are implemented.

\section{ACKNOWLEDGEMENTS}

Special thanks are expressed to Miss Srijanya, Miss Kednapat Sriphairoj, Miss Anyalak Wachirachaikarn, Mr. $\mathrm{Ha}$ Phuoc Hung who are studying Ph.D. programs in Fish Genetic Laboratory of Kasetsart University in Thailand for their assistances on laboratory work. Special thanks also to Dr. Huitong Zhou (Gene-Marker Laboratory, Lincoln University, New Zealand) for proofreading and suggestions for this research.

\section{REFERENCES}

Bandelt, H.J., 1995. Combinat ion of data in phylogenetic analysis. Plant Syst. Evol., 9: 355-361.

Bandlet, H.J., P. Forster and A. Rohl, 1999. Median joining networks for inferring intraspecific phylogenies. Mol. Biol. Evol., 16: 37-48.

Chaiwatanasin, W. and S. Chantsavang, 2002. Genetic Diversit $y$ of Native Pig in Thailand using microsatellite analysis. Kasetsart J. Nat. Sci., 36: 133-137.

Dadi, H., M. Tibbo, Y. Takahashi, K. Nomura and H. Hanada et al., 2008. Microsatellite analysis reveals high genet ic diversit y but low genetic structure in Ethiopian indigenous cattle populations. J. Anim. Genet., 39: 425-431.

Efron, B., E. Halloran and S. Holmes, 1996. Bootstrap confidence levels for phylogenet ic trees. Proc. Nat. Acad. Sci., 93: 7085-7090.

Excoffier, L.L.G. and S. Schneider, 2005. Arlequin ver. 3.0: An integrated software package for population genetics data analysis. Evol. Bioinform. Online, 1: $47-50$.

Falush, D., M. Stephens and J.K. Pritchard, 2003. Inference of population structure: Extensions to linked loci and correlated allele frequencies. Genetics, 164: 1567-1587.

Fan, B., Z.G. Wang, Y.J. Li, X.L. Zhao and B. Liu et al., 2002. Genetic variation analysis wit hin and among Chinese indigenous swine populations using microsatellite markers. Anim. Genet., 33: 422-427.

Fang, M., X. Hu, T. Jiang, M. Braunsweig and L. Hu et al., 2005. The phylogeny of Chinese Indigenous pig breeds inferred from microsatellite markers. Anim. Genet., 36: 7-13.
Felsenstein, J., 1993. PHYLIP (phylogeny inference package) version 3.6. Distribution by the author. Department of Genetics, University of Washington, Seattle.

Haig, S.M., 1998. Molecular contributions to conservation. Ecology, 79: 413-425.

Kazue, T., 1974. Morphological and serological studies on the native pigs in Thailand. Rep. Soc. Res. Native Livestock, 6: 181-183.

Kim, T.H., K.S.B. Kim, H. Choi, D.H. Yoon and G.W. Jang et al., 2005. Genetic structure of pig breeds from Korea and China using microsatellite loci analysis. J. Anim. Sci., 83: 2255-2263.

Kimura, M. and J.F. Crow, 1964. The number of alleles that can be maintained in a finite population. Genetics, 49: $725-738$.

Laval, G., N. Iannuccelli, C. Legault, D. Milan and M.A.M. Groenen et al., 2000. Genetic diversity of eleven European pig breeds. Genet. Select. Evolut., 32: 187-203.

Lemus-Flores, C., R. Ulloa-Arvizu, M. Ramos-Kuri, F.J. Estrada and R.A. Alonro et al., 2001. Genetic analysis of Mexican hairless pig population. J. Anim. Sci., 79: 3021-3026.

Levene, H., 1949. On a matching problem arising in genetics. Ann. Math. Stay, 20: 91-94.

Li, S.J., S.H. Yang, S.H. Zhao, B. Fan and M. Yu et al., 2004. Genetic diversity analyses of 10 indigenous Chinese pig populations based on 20 microsatellites. J. Anim. Sci., 82: 368-374.

Martin-Burriel, I., E. Garcia-Muro and P. Zaragoza, 1999. Genetic diversity analysis of six Spanish native cattle breeds using microsatellites. Anim. Genet, 30: 177-182.

Martinez, A.M., J.V. Delgado, A. Rodero and J.L.Vega-Pla, 2000. Genetic structure of the Iberian pig breed using microsatellites. Anim. Genet., 31: 295-301.

Murphy, T. and C. Tisdell, 1995. Trends in the Thai livestock industry, animal health implicatons and Thailand's development: An introduction. Research Reports and Papers in Animal Health Economics No. 8. Brisbane, Australia, Department of Economics, University of Queensland.

Nei, M., 1972. Genetic distance between populations. Am. Naturalist, 106: 283-292.

Nei, M., 1983. Estimat ion of fixation indices and gene diversities. Ann. Hum. Genet, 47: 253-259.

Pritchard, J.K., M. Stephens and P. Donnelly, 2000. Inference of population structure using multilocus genotype data. Genetics, 155: 945-959. 
Raymond, M. and F. Rousset, 1995. GENEPOP (Version 1.2): Population genetics software for exact tests and ecumenicism. J. Hered., 86: 248-249.

Ruane, J., 1999. A critical review of the values of genetic distance studies in conservation of animal genetic resources. J. Anim. Breed. Genet., 159: 699-713.

Sneath, P.H.A. andR.R. Sokal, 1973. Numerical Taxonomy. The Principles and Practice of Numerical Classification. W.H. Freeman, San Francisco, CA. Sokal.

Tanaka, K., L. Waxman and A.L. Goldberg, 1983. ATP serves two distinct roles ubiquitin. J. Cell Bid., 96: 1580-1585.

Templeton, A.R., K.A. Crandall and C.F. Sing, 1992. A cladistic analysis of phenotypic associations with haplotypes inferred from restriction endonuclease mapping and DNA sequence data. III. Cladogram Estimation. Genet., 132: 619-633.

Thirstrup, J.P., C. Pertoldi and V. Loeschcke, 2008. Genetic analysis, breed assignment and conservation priorities of three native Danish horse breeds. Anim. Genet., 39: 496-505.

Thuy, N.T.D., E. Melchinger-Wild, A.W. Kuss, N.V. Cuong and H. Bartenschlager et al., 2006. Comparison of Vietnamese and European pig breeds using microsatellites. J. Anim. Sci., 84: 2601-2608.
Tisdell, C. and S. Harrison, 1997. Livestock, the Environment and Sustainable Development with Illustrations of Issues from Thailand. In: Development that lasts, Roy, K.C., H.C. Blomqvist and I. Hossain (Eds.). New Age, New Delhi, pp: $190-200$.

Watanobe, T., N. Okumura, N. Ishiguro, M. Nakano and A. Matsui et al., 1999. Genetic relationship and distribution of the Japanese wild boar (Sus scrofa leucomystax) and Ryukyu wild boar (Sus scrofaryukiuanus) analyzed by mitochondrial DNA. Mol. Ecol., 8: 1509-1512.

Weir, B.S. and C.C. Cockerham, 1984. Estimating F-statistics for the analysis of population structure. Evolution, 38: 1358-1370.

Wright, S., 1965. The interpretation of population structure by F-statistics with special regard to systems of mating. Evolution, 19: 395-420.

Yang, S.L., Z.G. Wang, B. Liu, G.X. Zhang and S.H. Zhao et al., 2003. Genetic variation and relationships of eighteen Chinese indigenous pig breeds. Genet. Sel. Evol., 35: 657-671.

Yeh, F.C., R.C. Yang, T.B. Boyle, Z.H. Ye and J.X. Mao, 1997. POPGENE, The user-friendly shareware for populat ion genetic analysis. Molecular Biology and Biotechnology Centre, University of Alberta, Canada. 